\title{
Outstanding Thermal Conductivity of Single Atomic Layer Isotope-Modified Boron Nitride
}

\author{
Qiran Cai®, ${ }^{1}$ Declan Scullion, ${ }^{2}$ Wei Gan, ${ }^{1}$ Alexey Falin, ${ }^{1}$ Pavel Cizek, ${ }^{1}$ Song Liu, ${ }^{3}$ James H. Edgar, ${ }^{3}$ Rong Liu, ${ }^{4}$ \\ Bruce C. C. Cowie ${ }^{5},{ }^{5}$ Elton J. G. Santos ${ }^{6},{ }^{6}$ and Lu Hua Li ${ }^{1}{ }^{1, *}$ \\ ${ }^{1}$ Institute for Frontier Materials, Deakin University, Waurn Ponds Campus, Waurn Ponds VIC 3216, Australia \\ ${ }^{2}$ School of Mathematics and Physics, Queen's University Belfast, Belfast BT7 1NN, United Kingdom \\ ${ }^{3}$ Tim Taylor Department of Chemical Engineering, Kansas State University, Manhattan, Kansas 66506, USA \\ ${ }^{4}$ SIMS Facility, Office of the Deputy-Vice Chancellor (Research and Development), Western Sydney University, \\ Penrith NSW 2751, Australia \\ ${ }^{5}$ Australian Synchrotron, 800 Blackburn Road, Clayton VIC 3168, Australia \\ ${ }^{6}$ School of Physics and Astronomy, The University of Edinburgh, Edinburgh EH9 3FD, United Kingdom
}

(Received 23 February 2020; accepted 31 July 2020; published 21 August 2020; corrected 18 September 2020)

\begin{abstract}
Materials with high thermal conductivities $(\kappa)$ are valuable to solve the challenge of waste heat dissipation in highly integrated and miniaturized modern devices. Herein, we report the first synthesis of atomically thin isotopically pure hexagonal boron nitride $(\mathrm{BN})$ and its one of the highest $\kappa$ among all semiconductors and electric insulators. Single atomic layer $(1 L) \mathrm{BN}$ enriched with ${ }^{11} \mathrm{~B}$ has a $\kappa$ up to $1009 \mathrm{~W} / \mathrm{mK}$ at room temperature. We find that the isotope engineering mainly suppresses the out-of-plane optical (ZO) phonon scatterings in $\mathrm{BN}$, which subsequently reduces acoustic-optical scatterings between $\mathrm{ZO}$ and transverse acoustic (TA) and longitudinal acoustic phonons. On the other hand, reducing the thickness to a single atomic layer diminishes the interlayer interactions and hence umklapp scatterings of the out-of-plane acoustic (ZA) phonons, though this thickness-induced $\kappa$ enhancement is not as dramatic as that in naturally occurring BN. With many of its unique properties, atomically thin monoisotopic $\mathrm{BN}$ is promising on heat management in van der Waals devices and future flexible electronics. The isotope engineering of atomically thin $\mathrm{BN}$ may also open up other appealing applications and opportunities in 2D materials yet to be explored.
\end{abstract}

DOI: 10.1103/PhysRevLett.125.085902

A dramatic temperature rise can occur locally at the so-called "hot spot" in highly integrated and miniaturized devices, including microprocessor and circuit packages, light-emitting diodes, lasers, and high-power radio frequency transmitters. Eliminating waste heat limits the performance, reliability, and longevity of many modern devices. Materials with outstanding thermal conductivities $(\kappa)$ are one of the key solutions to this challenge. While diamond and graphite are the only two traditional materials with $\kappa$ above $1000 \mathrm{~W} / \mathrm{mK}$ at room temperature, emerging materials such as carbon nanotubes [1], graphene [2], and recently discovered cubic boron arsenide ( $c \mathrm{BAs})$ $(\sim 1000 \mathrm{~W} / \mathrm{mK})$ [3-5] are also excellent thermal conductors. However, electrically conductive carbon materials are not suitable in direct contact with electronic devices due to the potential for short circuiting. Although diamond and $c \mathrm{BAs}$ have the potential for high-power electronics, they are unsuitable for flexible electronic devices and new twodimensional (2D) van der Waals (vdW) structures.

Distinct from their carbon counterparts, all boron nitride (BN) materials, including single-wall nanotubes and single atomic layer or monolayer $(1 L) \mathrm{BN}$ are electric insulators and hence better candidates for waste heat dissipation, for example, in electronic devices. Bulk cubic $(c)$ and hexagonal $(h) \mathrm{BN}$ crystals are good thermal conductors with $\kappa$ of $\sim 690$ and $420 \mathrm{~W} / \mathrm{mK}$ at room temperature, respectively [6]. Recently, we reported that high-quality and surface-clean $1 L h \mathrm{BN}$ had a $\kappa$ of $751 \pm 340 \mathrm{~W} / \mathrm{mK}$ [7]. This $\kappa$ increase with reduced thickness down to the atomic level was due to a decrease in the number of phonon branches and states available for umklapp scattering with less interlayer interaction. Defects, grain boundaries, and surface contaminations, nevertheless, could adversely affect the thermal conduction of atomically thin BN [8-12].

Isotope engineering affects many fundamental properties of a solid, e.g., lattice parameter, disordering, elastic constant, vibration, band structure and transition, exciton, polariton dispersion, and scattering. It, in turn, gives rise to appealing phenomena and applications, including the elevation of superconducting transition temperature, improvement in the lifetime of organic light-emitting diodes (OLED), optical fibers with higher speed, precise and accurate quantification of proteomes, and ultratrace environmental analysis [13-15]. Naturally occurring BN (NatBN) contains a relatively high percentage of two stable boron isotopes: $19.9 \%{ }^{10} \mathrm{~B}$ and $80.1 \%{ }^{11} \mathrm{~B}$; while carbon 
(C) normally consists of $98.9 \%{ }^{12} \mathrm{C}$ and only $1.1 \%{ }^{13} \mathrm{C}$. The phonon energy, electronic bandgap, and electron density distribution of $h \mathrm{BN}$ could be varied by isotope engineering [16]. Isotope enriched $h \mathrm{BN}$ greatly increased the polariton lifetime [17]. In addition, ${ }^{10} \mathrm{~B}$ is one of the best neutron absorbers and used widely in radiation shielding, nuclear reactivity control, and neutron capture therapy for tumor treatment $[18,19]$. Replacing ${ }^{10} \mathrm{~B}$ by ${ }^{11} \mathrm{~B}$, on the other hand, prevents electronic devices from data loss or single-event upset caused by cosmic rays or their generation of ionizing particles.

Reducing isotopic disorder also increases thermal conductivity. The in-plane $\kappa$ of isotopically pure ${ }^{12} \mathrm{C}$ graphene is $36 \%$ and $\sim 100 \%$ higher than that of naturally occurring graphene and graphite, respectively [20]. The $\kappa$ of $1 L$ isotopically pure ${ }^{100} \mathrm{MoS}_{2}$ were $61.6 \mathrm{~W} / \mathrm{mK}$, larger than the $40.8 \mathrm{~W} / \mathrm{mK}$ of $1 L{ }^{\mathrm{Nat}} \mathrm{MoS}_{2}$ [21]. Note that chemical vapor deposition (CVD) was used to synthesize these monoisotopic graphene and $\mathrm{MoS}_{2}$. In terms of $\mathrm{BN}$, the room temperature $\kappa$ of bulk ${ }^{10} \mathrm{BN}$ crystals was $585 \mathrm{~W} / \mathrm{mK}, \sim 39 \%$ higher than that of bulk NatBN [22]. The effect of isotopic impurity on the $\kappa$ of BN nanotubes was also studied: $310 \mathrm{~W} / \mathrm{mK}$ for ${ }^{11} \mathrm{BN}$ nanotubes, much larger than the $200 \mathrm{~W} / \mathrm{mK}$ of ${ }^{\mathrm{Nat}} \mathrm{BN}$ nanotubes as a control [23]. Very recently, an ultrahigh $\kappa$ of $1600 \mathrm{~W} / \mathrm{mK}$ was achieved from isotopically enriched $c \mathrm{BN}, \sim 190 \%$ higher than that of natural occurring $c \mathrm{BN}$ [24]. However, there has been no report on the synthesis of atomically thin monoisotopic BN, let alone measurement of its $\kappa$, though a 25\%-36\% enhancement in $\kappa$ was theoretically predicted from $1 L$ isotopically pure $\mathrm{BN}$ compared to that of $1 L^{\mathrm{Nat}} \mathrm{BN}$ [25-28].

In this work, we successfully produced atomically thin isotopically pure ${ }^{10} \mathrm{BN}$ and ${ }^{11} \mathrm{BN}$ for the first time, and their intrinsic in-plane thermal conductivities could be determined due to their high quality and clean surface. Based on optothermal Raman measurements, the $\kappa$ of $1 L$ ${ }^{11} \mathrm{BN}$ and ${ }^{10} \mathrm{BN}$ were $1009 \pm 313$ and $958 \pm 355 \mathrm{~W} / \mathrm{mK}$, respectively. These values were $\sim 34 \%$ and $\sim 140 \%$ larger than those of $1 L$ and bulk NatBN, respectively. Density functional theory (DFT) simulations were used to gain insights into the isotope effect. This study may also give rise to new possibilities in many other applications, e.g., multifunctional metal-matrix nanocomposites for radiation shielding and new cancer treatment $[18,19]$.

High-quality and surface-clean atomically thin isotopically pure BN sheets were mechanically exfoliated from bulk crystals grown by the nickel-chromium solvent method $[16,29]$. According to secondary ion mass spectrometry (SIMS), these bulk crystals contained $99.2 \%$ and $99.9 \%{ }^{10} \mathrm{~B}$ and ${ }^{11} \mathrm{~B}$, respectively, close to the previously reported values [16]. Naturally occurring nitrogen has $>99.6 \%{ }^{14} \mathrm{~N}$, and thus can be considered as isotopically pure. The atomically thin ${ }^{10} \mathrm{BN}$ and ${ }^{11} \mathrm{BN}$ sheets were directly exfoliated and suspended over prefabricated
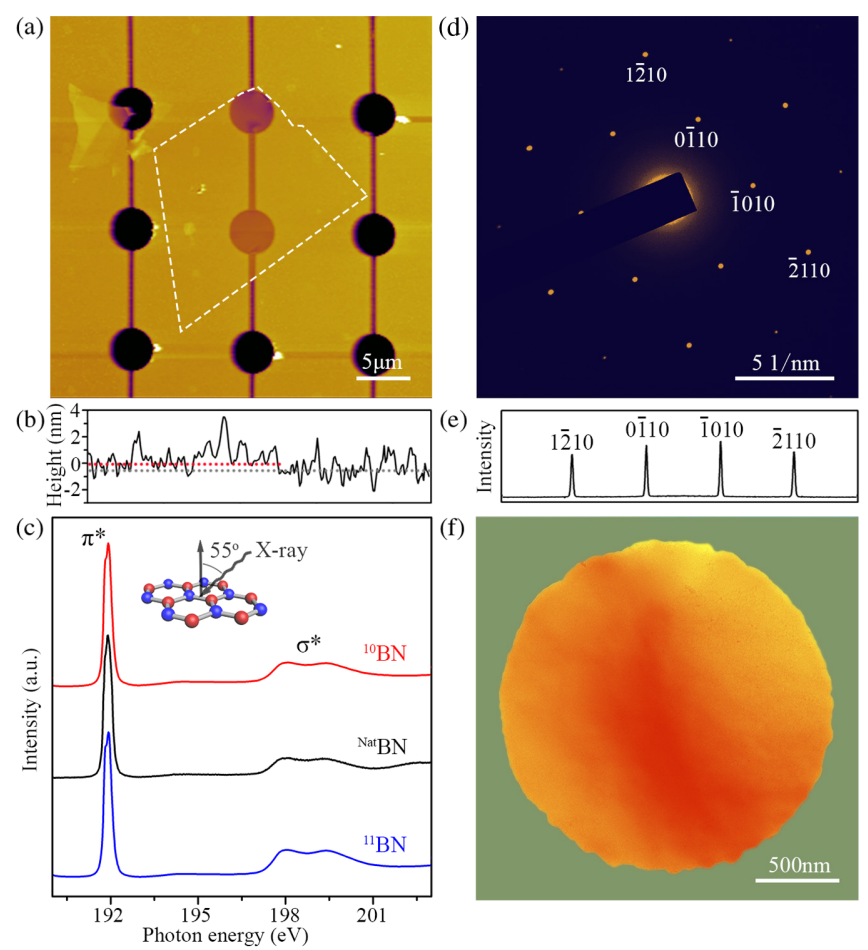

FIG. 1. (a) AFM image of a mechanically exfoliated $1 L{ }^{10} \mathrm{BN}$ suspended over $\mathrm{Au} / \mathrm{Si}$ substrate with microwells and trenches. (b) AFM height of the $1 L$ sample. (c) NEXAFS spectra in the B $K$-edge region of isotopically pure and natural $\mathrm{BN}$ at the incidence of $55^{\circ}$. (d) Selected area electron diffraction of a suspended $1 L{ }^{10} \mathrm{BN}$. (e) The corresponding diffraction spot intensities and (f) the DF-TEM image.

microwells $(3.8 \mu \mathrm{m}$ in diameter) connected by narrow trenches (200 nm in width) in $80 \mathrm{~nm}$ gold-coated $\mathrm{Si}$ substrates $(\mathrm{Au} / \mathrm{Si})$ without polymer-assisted transfer process. The Au films served as heat sinks during measurements; the trench acted as vents to avoid strain induced to the atomically thin materials due to volume change of the air trapped in the microwells during heating.

Figures 1(a) and 1(b) show the atomic force microscopy (AFM) image and height trace of a suspended $1 L{ }^{10} \mathrm{BN}$ with a thickness of $0.50 \mathrm{~nm}$. More samples and AFM images are shown in the Supplemental Material, Fig. S1 [30]. The chemical composition, crystal structure, and quality of the isotopically pure samples were probed by near-edge $\mathrm{x}$-ray absorption fine structure (NEXAFS) spectroscopy, and compared with those of a single crystal ${ }^{N a t} \mathrm{BN}$ synthesized by the high pressure Ba-BN solvent method [Fig. 1(c)] [31]. Sharp $\pi^{*}$ resonances at $192.0 \mathrm{eV}$ corresponding to $1 \mathrm{~s}$ core electron transitions to the unoccupied antibonding orbitals of $\mathrm{B}$ atoms $\mathrm{sp}^{2}$ bond to three $\mathrm{N}$ atoms were observed from all samples, verifying their hexagonal crystal structure. No satellite peaks caused by other chemical environments were present, suggesting high chemical purities of the isotopically pure samples $[32,33]$. These results are in line with the previous finding 
that the ${ }^{10} \mathrm{BN}$ and ${ }^{11} \mathrm{BN}$ crystals were free of defects in the areas of tens of microns [22].

Transmission electron microscope (TEM) was used to detect whether the exfoliated atomically thin sheets contained any grain boundary. Figure 1(d) shows a typical electron diffraction pattern from the entire suspended area of a $1 L{ }^{10} \mathrm{BN}$ on a perforated silicon nitride $\left(\mathrm{SiN}_{x}\right)$ TEM grid with $2 \mu \mathrm{m}$ holes. The diffraction pattern consisted of single sets of diffraction dots with sixfold symmetry. The intensity profile of the (12 10$),(0 \overline{1} 10),(\overline{1} 010)$, and $(\overline{2} 110)$ diffraction reflections is plotted in Fig. 1(e), which can be compared with those of $2 L$ and few-layer samples shown in the Supplemental Material, Fig. S2 [30]. The dark field (DF)-TEM image also revealed a monocrystalline nature without grain boundaries [Fig. 1(f)]. The TEM results of a $1 \mathrm{~L}{ }^{11} \mathrm{BN}$ are available in the Supplemental Material, Fig. S3 [30].

Figure 2(a) compares the Raman spectra of suspended $1-3 L$ and bulk ${ }^{10} \mathrm{BN}$, Nat $\mathrm{BN}$, and ${ }^{11} \mathrm{BN}$. Since the Raman frequency is inversely proportional to the square root of mean atomic mass, the bulk ${ }^{10} \mathrm{BN}$, NatBN, and ${ }^{11} \mathrm{BN}$ crystals showed $G$ bands centered at 1392.0, 1366.9, and $1358.0 \mathrm{~cm}^{-1}$, respectively. Reducing the thickness of suspended monoisotopic BN to the atomic scale barely changed their $G$ band Raman frequencies but lowered the peak intensities. A similar phenomenon on atomically thin ${ }^{N a t} \mathrm{BN}$ was reported and explained by us before [34,35].
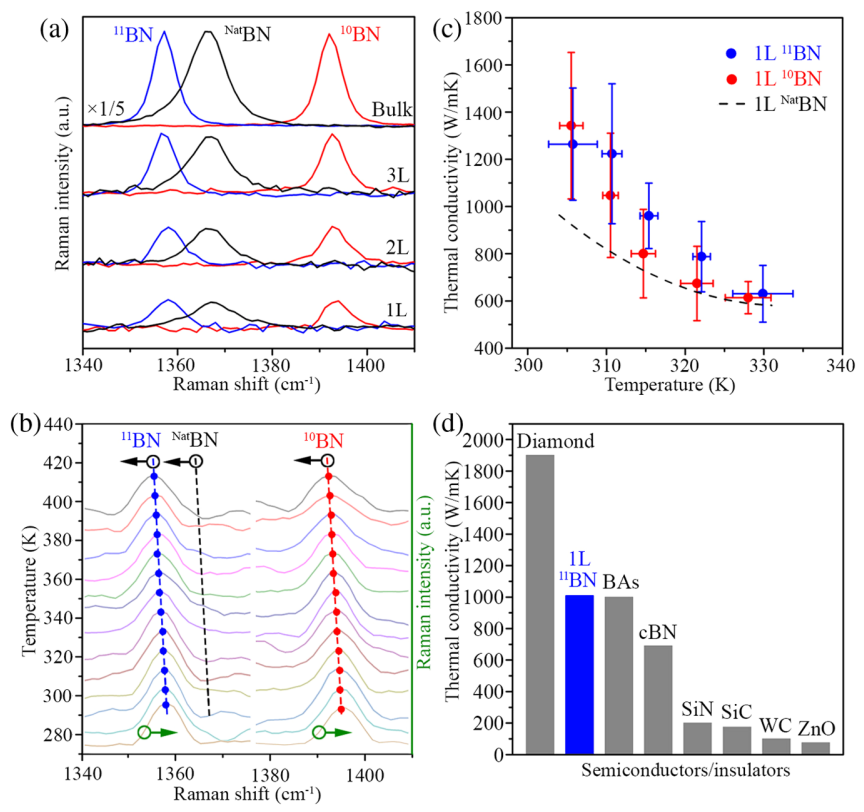

FIG. 2. (a) Comparison among Raman spectra of $1 L-3 L$ and bulk ${ }^{10} \mathrm{BN}$, NatBN, and ${ }^{11} \mathrm{BN}$; (b) temperature effect on the Raman $G$ band frequency of $1 L{ }^{10} \mathrm{BN}$, NatBN, and ${ }^{11} \mathrm{BN}$ sheets with corresponding fittings; (c) experimental $\kappa$ of $1 L{ }^{10} \mathrm{BN}$ (red) and ${ }^{11} \mathrm{BN}$ (blue) with standard deviations as a function of temperature, compared with that of $1 L{ }^{N a t B N}$ (black and dashed); (d) the comparison among $\kappa$ of representative semiconductors and insulators.
Because of mass disorder effects, the different isotope mass also affected the full width at half maximum (FWHM) of the $G$ bands of bulk ${ }^{10} \mathrm{BN}$, Nat $\mathrm{BN}$, and ${ }^{11} \mathrm{BN}$ crystals, i.e., $5.9,9.4$, and $5.6 \mathrm{~cm}^{-1}$, respectively [36]. The atomically thin sheets showed broader bandwidths, caused by stronger surface scattering influencing the vibrational excitation lifetime [34,35].

The $\kappa$ of atomically thin ${ }^{10} \mathrm{BN}$ and ${ }^{11} \mathrm{BN}$ was measured by optothermal Raman technique [2,7,10,21,37-40]. First, the Raman $G$ band frequency of the suspended $1 L{ }^{10} \mathrm{BN}$ and ${ }^{11} \mathrm{BN}$ as a function of temperature was determined using a hot plate with accurate temperature control $\left( \pm 0.1^{\circ} \mathrm{C}\right)$. In order to minimize laser heating, a small laser power of $\sim 1.5 \mathrm{~mW}$ was chosen. Figure 2(b) summarizes the Raman $G$ bands of a $1 L{ }^{10} \mathrm{BN}$ and ${ }^{11} \mathrm{BN}$ at $293-413 \mathrm{~K}$ with an interval of $10 \mathrm{~K}$ and the corresponding linear fittings, i.e., $\omega-\omega_{0}=\chi T$, where $\chi$ is the first-order temperature coefficient, and $\omega-\omega_{0}$ is the change of the $G$ band frequency due to temperature variation. The $\chi$ values of $1 L{ }^{10} \mathrm{BN}$ and ${ }^{11} \mathrm{BN}$ were $-0.0223 \pm 0.0018$ [red dashed line in Fig. 2(b)] and $-0.0220 \pm 0.0022$ (blue dashed line) $\mathrm{cm}^{-1} / \mathrm{K}$, respectively, quite similar to that of ${ }^{N a t B N}$, i.e., $-0.0223 \pm 0.0012 \mathrm{~cm}^{-1} / \mathrm{K}$ (black dashed line) [7]. Note that the volumetric thermal expansion of the Au/Si substrate during heating hardly affected these $\chi$ values due to the hanging down of suspended atomically thin $\mathrm{BN}$, releasing the strain induced by thermal expansion coefficients mismatch between $\mathrm{BN}$ and the substrate, as we described before [7].

The suspended $1 \mathrm{~L}{ }^{10} \mathrm{BN}$ and ${ }^{11} \mathrm{BN}$ sheets were then optically heated under different laser power $(4-10 \mathrm{~mW})$ to increase the local temperature $\left(T_{m}\right)$ (see Supplemental Material, Fig. S4 [30] for the Raman spectra). Given that the heat flux vector is along the radial direction away from the center of the suspended BN sheets and the phonon transport is diffusive, the thermal conductivity was calculated by

$$
\kappa=\frac{\ln \left(\frac{R}{r_{0}}\right)}{2 \pi t \frac{T_{m}-T_{a}}{Q-Q_{\text {air }}}} \alpha,
$$

where $R$ is the radius of the microwell $(1.9 \mu \mathrm{m}) ; r_{0}$ is the radius of the laser beam that was $0.32 \pm 0.01 \mu \mathrm{m}$ (see Supplemental Material, Fig. S5 [30]). Here, the laser beam radius $\left(r_{0}\right)$ was the position where the laser intensity decreases to $1 / e$ of its maximum intensity. The definition of $r_{0}$ at either $1 / e$ or $1 / e^{2}$ intensity does not affect the obtained value of thermal conductivity (see Supplemental Material [30]). $\alpha=0.97$ is the Gaussian profile factor of the laser beam; $t$ is the BN thickness; $T_{m}$ is the temperature measured by Raman; $T_{a}$ is the ambient temperature; $Q$ is the absorbed laser power $[20,38]$. The optical absorption of $1 L{ }^{10} \mathrm{BN}$ and ${ }^{11} \mathrm{BN}$ at $488 \mathrm{~nm}$ wavelength was determined by the difference in the measured laser power between empty and nearby $\mathrm{BN}$-covered holes of $\mathrm{SiN}_{x}$ 
grids (see Supplemental Material, Fig. S6 [30]). That is, $Q=P_{\text {empty }}-P_{\mathrm{BN}}$. There was no noticeable difference in the absorbance of $1 L{ }^{10} \mathrm{BN}$ and ${ }^{11} \mathrm{BN}$, and the averaged value was $(0.32 \pm 0.13) \%$, close to that of $1 L{ }^{\text {NatBN [7]. }}$ $Q_{\text {air }}$ is the heat loss in the air:

$$
Q_{\text {air }}=\int_{r_{0}}^{R} 2 \pi h\left(T-T_{a}\right) r d r+\pi r_{0}^{2} h\left(T_{m}-T_{a}\right),
$$

where $T$ is the temperature at radius $r ; h$ is the heat transfer coefficient of $h \mathrm{BN}$. In the case of small temperature variation between an object and the ambient, the quadratic expression for radiation can be simplified to the linearized sum of convective $\left(h_{c}\right)$ and radiative $\left(h_{r}\right)$ components to obtain the total heat transfer coefficient. That is, $h=h_{c}+h_{r}$, where $h_{c}=3475 \mathrm{~W} / \mathrm{m}^{2} \mathrm{~K}$ for $\mathrm{BN}$ sheets; $h_{r}=\varepsilon \sigma 4 T^{3} ; \varepsilon=0.8$ is the emissivity of $h \mathrm{BN}$; and $\sigma$ is the Stefan-Boltzmann constant with the value of $5.670376 \times 10^{-8} \mathrm{~W} / \mathrm{m}^{2} \mathrm{~K}^{4}[9]$.

The $\kappa$ of $1 L^{10} \mathrm{BN}$ and ${ }^{11} \mathrm{BN}$ as a function of temperature was calculated based on Eq. (1), and compared with that of $1 L^{\text {Nat } B N}$ from our previous study [Fig. 2(c)] [7]. The errors were calculated through the root sum square error propagation approach, where the temperature calibration by Raman, temperature resolution of the Raman measurements, and the uncertainty of the measured laser absorbance were considered. Because of the small temperature range and the uncertainty of the optothermal technique, we averaged the $\kappa$ values: $958 \pm 355$ and $1009 \pm 313 \mathrm{~W} / \mathrm{mK}$ for $1 L^{10} \mathrm{BN}$ and ${ }^{11} \mathrm{BN}$ near room temperature, respectively. The heat loss to air only accounted for about $1.8 \%$ of the total heat dissipation during laser heating, indicating the use of the emissivity of $h \mathrm{BN}$ did not affect the calculated $\kappa$ of atomically thin BN much. Note optical heating was coupled more strongly to diffusive phonons of higher frequency than ballistic phonons, and the temperature measured by the Raman method was the anharmonic scattering temperature between the zone-center or zone-boundary optical phonons and diffusive acoustic phonons $[39,41,42]$. In addition, the local nonequilibrium of phonon polarizations was ignored [43]. As a result, these Raman-deduced $\kappa$ values should be underestimated. Our results showed that the $\kappa$ of $1 L$ monoisotopic BN was about $34 \%$ and $140 \%$ higher than those of $1 \mathrm{~L}{ }^{N a t} \mathrm{BN}$ and bulk

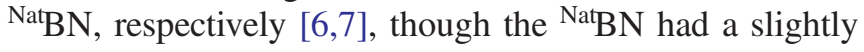
lower destiny of defects. Figure 2(d) compares the $\kappa$ of $1 L$ ${ }^{11} \mathrm{BN}$ with those of some representative semiconductors and insulators.

Theoretical calculations were used to comprehensively understand the isotope effects. In the ab initio calculations of the $\kappa$ of $1 L{ }^{10} \mathrm{BN}\left(99.2 \%{ }^{10} \mathrm{~B}\right),{ }^{\mathrm{Nat}} \mathrm{BN}$, and ${ }^{11} \mathrm{BN}(99.9 \%$ ${ }^{11} \mathrm{~B}$ ), phonon-phonon, isotope, and boundary scatterings were taken into account. The boundary scattering rate was calculated as $v_{g} / L$, where $v_{g}$ is the group velocity of the phonons, and $L$ is the boundary length. Isotope mixing caused isotope scattering and shortened phonon mean free path $(\lambda)$. In excellent agreement with our experimental results, the $99.9 \%{ }^{11} \mathrm{BN}$ had a slightly higher $\kappa$ than the $99.2 \%{ }^{10} \mathrm{BN}$ at $4 \mu \mathrm{m}$ length close to the experimental sample size [Fig. 3(a)], revealing that the higher isotope purity in ${ }^{11} \mathrm{BN}$ was the main cause of the slightly higher $\kappa$. Figure 3(b) shows the theoretical accumulative $\kappa$ as a function of phonon frequency, and for comparison purpose, the phonon dispersions of $1 L{ }^{10} \mathrm{BN}$ and ${ }^{11} \mathrm{BN}$ are displayed in Fig. 3(c). Apparently, the out-of-plane acoustic phonons (ZA) contributed to most of $\kappa$ in all BN sheets, consistent with our previous study [7]. Interestingly, $1 L^{10} \mathrm{BN}$, NatBN, and ${ }^{11} \mathrm{BN}$ showed almost no difference in the accumulative $\kappa$ at phonon frequency lower than $320 \mathrm{~cm}^{-1}$, indicating that the isotope mixing had a small influence on the ZA phonons [Fig. 3(c)]. In the region of $320-600 \mathrm{~cm}^{-1}$ [highlighted in light gray in Figs. 3(b) and 3(c)], the accumulative $\kappa$ started to split between $1 L$ naturally occurring and monoisotopic BN. This means that the
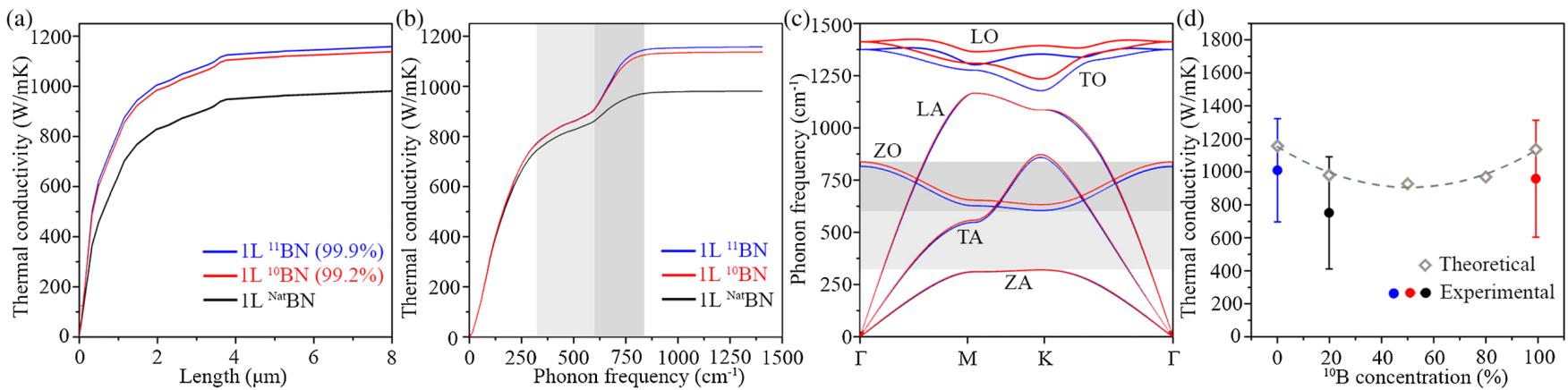

FIG. 3. (a) Theoretical cumulative $\kappa$ of $1 L^{10} \mathrm{BN}(99.2 \%),{ }^{11} \mathrm{BN}(99.9 \%)$, and ${ }^{N a t} \mathrm{BN}$ as a function of sample length. (b) Theoretical cumulative $\kappa$ of the same three materials as a function of phonon frequency, and the two phonon-frequency regions where $\kappa$ splits from different phonon contributions (see text for details) between $1 L$ naturally occurring and monoisotopic BN are highlighted. (c) Phonon dispersion of $1 L{ }^{10} \mathrm{BN}$ (red) and ${ }^{11} \mathrm{BN}$ (blue) with the same phonon-frequency regions of $\kappa$ divergence highlighted. (d) Theoretical (open rhombus) and experimental (solid dots with standard deviations) $\kappa$ of $1 \mathrm{~L} \mathrm{BN}$ as a function of ${ }^{10} \mathrm{~B}$ concentration (i.e., $0.1 \%, 19.9 \%, 50 \%$, $80 \%$, and $99.2 \%$ ), and corresponding parabolic fitting (dashed line). 
isotopic purification decreased the scattering of transverse acoustic (TA) and longitudinal acoustic (LA) phonons. Nevertheless, the most significant deviation in $\kappa$ happened at $\sim 600-800 \mathrm{~cm}^{-1}$, corresponding well to the contribution of the out-of-plane optical phonons (ZO) [highlighted in dark gray in Figs. 3(b) and 3(c)]. This suggests that the ZO phonon-isotope scatterings play an important role in the thermal conductivity of NatBN. Optical phonons barely contribute by themselves to thermal conductivity in bulk materials $[44,45]$; however, as dimensionality is reduced, an important scattering channel for acoustic phonons is mediated through their optical counterparts. We observed that the majority of the difference in $\kappa$ between $1 L$ naturally occurring and monoisotopic $\mathrm{BN}$ was caused by a strong acoustic-optical phonon scattering between $\mathrm{ZO}$ and TA/LA phonons due to isotope mixing [45]. Figure 3(d) compares the $\kappa$ of $1 L \mathrm{BN}$ with different ${ }^{10} \mathrm{~B}$ concentrations, which followed a parabolic trend. For isotope scattering, $\lambda \propto g^{-1} T^{-4}$, where $g=\sum_{i} C_{i}$ $\left[\left(M_{i}^{2}-\left(\sum C_{i} M_{i}\right)\right) /\left(\sum C_{i} M_{i}\right)^{2}\right]^{2} ; T$ is the temperature; $C_{i}$ and $M_{i}$ are the concentration and mass of isotope atoms, respectively. $g$ reached its maximum at about $50 \%$ ${ }^{10} \mathrm{~B}$, resulting in the minimum $\lambda$ and hence $\kappa$. Our theoretical $\kappa$ values were larger than the experimental values and other theoretical predictions based on classical potentials (see Supplemental Material, Table S1 [30]), and this could be due to the local density approximation (LDA). LDA is well known to over bind systems, leading to overestimations of phonon frequencies and consequently thermal conductivity [46].

We also measured the $\kappa$ of few-layer monoisotopic BN using the same procedure. They had smaller $\kappa$ values than those of the corresponding monolayers: $880 \pm 174$ and $833 \pm 209 \mathrm{~W} / \mathrm{mK}$ for $2 L$ and $3 L^{10} \mathrm{BN}$, and $930 \pm 204$ and $902 \pm 193 \mathrm{~W} / \mathrm{mK}$ for $2 L$ and $3 L{ }^{11} \mathrm{BN}$, respectively, as shown in Fig. S7 of Ref. [30]. The smaller $\kappa$ values were attributed to the increased umklapp scattering of ZA phonons [7]. It is worth noting that the differences in $\kappa$ among $1 L-3 L$ monoisotopic $\mathrm{BN}$ (i.e., ${ }^{10} \mathrm{BN}: 1: 0.92: 0.87$; $\left.{ }^{11} \mathrm{BN}: 1: 0.92: 0.89\right)$ were smaller than those among $1 L-3 L$ NatBN $(1: 0.86: 0.80)$ [7]. This can be explained by the larger contribution ratio of the ZA phonons to the overall thermal conductivity of NatBN than monoisotopic $\mathrm{BN}$, because as aforementioned, reducing isotope mixing does not affect the ZA phonons much but significantly increases the contribution of TA/LA phonons to the thermal conductivity [Fig. 3(b)]. The additional layers in $2 L-3 L \mathrm{BN}$ have a much larger influence on the ZA phonon scatterings than TA and LA phonons (Fig. S8 [30]). As a result, the increased umklapp scatterings of ZA phonons due to the additional layers give rise to more prominent decreases in the thermal conductivity of few-layer NatBN than monoisotopic BN.

In summary, high-quality and suspended atomically thin isotopically pure $\mathrm{BN}$ sheets were produced by mechanical exfoliation, and their intrinsic in-plane thermal conductivities were measured by the optothermal Raman technique: $958 \pm 355$ and $1009 \pm 313 \mathrm{~W} / \mathrm{mK}$ for $1 L^{10} \mathrm{BN}$ and ${ }^{11} \mathrm{BN}$ at close-to room temperature, respectively. These values were about $34 \%$ and $140 \%$ larger than those of $1 \mathrm{~L}$ and bulk NatBN, respectively, attributed to (i) the longer mean free path of phonons mainly due to less $\mathrm{ZO}$ phonon-isotope scattering and subsequent reduced acoustic-optical scatterings between $\mathrm{ZO}$ and TA/LA phonons; (ii) decreased phonon umklapp scatterings in atomically thin samples caused by less interlayer interactions and hence reduced phonon branches. With its layered structure, low density, wide band gap, excellent mechanical flexibility and strength, good chemical and thermal stability, atomically thin monoisotopic $\mathrm{BN}$ is promising on heat management in $\mathrm{vdW}$ devices and flexible electronics. This study not only deepens the fundamental understanding of isotope effect on 2D thermal conductivity but also forms the basis for further research and applications of isotopically engineered $2 \mathrm{D}$ materials.

L. H. L. thanks Professor Takashi Taniguchi and Dr. Kenji Watanabe from National Institute for Materials Science, Japan for providing NathBN single crystals for comparison; L. H.L. thanks the financial support from Australian Research Council (ARC) via Discovery Early Career Researcher Award (DE160100796). Q.C. acknowledges ADPRF from Deakin University. E. J. G. S. acknowledges the use of computational resources from the UK Materials and Molecular Modelling Hub for access to THOMAS supercluster, which is partially funded by EPSRC (EP/P020194/1) and the Cirrus UK National Tier-2 HPC Service at EPCC funded by the University of Edinburgh and EPSRC (EP/P020267/1) under Contract No. ec019. The Queen's Fellow Award through Grant No. M8407MPH, and the Department for the Economy (USI 097) are also acknowledged. Support from the Materials Engineering and Processing program of the National Science Foundation, Grant No. CMMI 1538127, and the II-VI Foundation for monoisotopic $h \mathrm{BN}$ crystal growth is greatly appreciated. Part of the work was done at the Melbourne Centre for Nanofabrication $(\mathrm{MCN})$ in the Victorian Node of the Australian National Fabrication Facility (ANFF) and on the soft x-ray beam line at the Australian Synchrotron, Victoria, Australia.

*luhua.li@deakin.edu.au

[1] S. Berber, Y. K. Kwon, and D. Tomanek, Unusually High Thermal Conductivity of Carbon Nanotubes, Phys. Rev. Lett. 84, 4613 (2000).

[2] A. A. Balandin, S. Ghosh, W. Bao, I. Calizo, D. Teweldebrhan, F. Miao, and C. N. Lau, Superior thermal 
conductivity of single-layer graphene, Nano Lett. 8, 902 (2008).

[3] S. Li, Q. Zheng, Y. Lv, X. Liu, X. Wang, P. Y. Huang, D. G. Cahill, and B. Lv, High thermal conductivity in cubic boron arsenide crystals, Science 361, 579 (2018).

[4] F. Tian et al., Unusual high thermal conductivity in boron arsenide bulk crystals, Science 361, 582 (2018).

[5] J. S. Kang, M. Li, H. Wu, H. Nguyen, and Y. Hu, Experimental observation of high thermal conductivity in boron arsenide, Science 361, 575 (2018).

[6] P. Q. Jiang, X. Qian, R. G. Yang, and L. Lindsay, Anisotropic thermal transport in bulk hexagonal boron nitride, Phys. Rev. Mater. 2, 064005 (2018).

[7] Q. Cai, D. Scullion, W. Gan, A. Falin, S. Zhang, K. Watanabe, T. Taniguchi, Y. Chen, E. J. G. Santos, and L. Hua Li, High thermal conductivity of high-quality monolayer boron nitride and its thermal expansion, Sci. Adv. 5, eaav0129 (2019).

[8] I. Jo, M. T. Pettes, J. Kim, K. Watanabe, T. Taniguchi, Z. Yao, and L. Shi, Thermal conductivity and phonon transport in suspended few-layer hexagonal boron nitride, Nano Lett. 13, 550 (2013).

[9] M. T. Alam, M. S. Bresnehan, J. A. Robinson, and M. A. Haque, Thermal conductivity of ultra-thin chemical vapor deposited hexagonal boron nitride films, Appl. Phys. Lett. 104, 013113 (2014).

[10] H. Q. Zhou et al., High thermal conductivity of suspended few-layer hexagonal boron nitride sheets, Nano Res. 7, 1232 (2014).

[11] C. Wang, J. Guo, L. Dong, A. Aiyiti, X. Xu, and B. Li, Superior thermal conductivity in suspended bilayer hexagonal boron nitride, Sci. Rep. 6, 25334 (2016).

[12] Z. Lin, C. Liu, and Y. Chai, High thermally conductive and electrically insulating 2D boron nitride nanosheet for efficient heat dissipation of high-power transistors, 2D Mater. 3, 041009 (2016)

[13] C.C. Tong and K. C. Hwang, Enhancement of OLED efficiencies and high-voltage stabilities of light-emitting materials by deuteration, J. Phys. Chem. C 111, 3490 (2007).

[14] S. Konegger-Kappel and T. Prohaska, Spatially resolved analysis of plutonium isotopic signatures in environmental particle samples by laser ablation-MC-ICP-MS, Anal. Bioanal. Chem. 408, 431 (2016).

[15] X. Shen et al., Ionstar enables high-precision, low-missingdata proteomics quantification in large biological cohorts, Proc. Natl. Acad. Sci. U.S.A. 115, E4767 (2018).

[16] T. Q. P. Vuong, S. Liu, A. Van der Lee, R. Cuscó, L. Artús, T. Michel, P. Valvin, J. H. Edgar, G. Cassabois, and B. Gil, Isotope engineering of van der Waals interactions in hexagonal boron nitride, Nat. Mater. 17, 152 (2018).

[17] A. J. Giles et al., Ultralow-loss polaritons in isotopically pure boron nitride, Nat. Mater. 17, 134 (2018).

[18] A. Akkaş, A. Tugrul, O. Addemir, M. Marşoğlu, B. Ağacan, and B. Büyük, Radiation shielding effect of boron carbide aluminum metal matrix composite, Acta Phys. Pol. A 127, 947 (2015).

[19] R. F. Barth, A. H. Soloway, R. G. Fairchild, and R. M. Brugger, Boron neutron capture therapy for cancer, Realities and prospects, Cancer 70, 2995 (1992).
[20] S. Chen, Q. Wu, C. Mishra, J. Kang, H. Zhang, K. Cho, W. Cai, A. A. Balandin, and R. S. Ruoff, Thermal conductivity of isotopically modified graphene, Nat. Mater. 11, 203 (2012).

[21] X. Li et al., Isotope-engineering the thermal conductivity of two-dimensional $\mathrm{MoS}_{2}$, ACS Nano 13, 2481 (2019).

[22] C. Yuan, J. Li, L. Lindsay, D. Cherns, J. W. Pomeroy, S. Liu, J.H. Edgar, and M. Kuball, Modulating the thermal conductivity in hexagonal boron nitride via controlled boron isotope concentration, Communications in Physics 2, 43 (2019).

[23] C. Chang, A. Fennimore, A. Afanasiev, D. Okawa, T. Ikuno, H. Garcia, D. Li, A. Majumdar, and A. Zettl, Isotope Effect on the Thermal Conductivity of Boron Nitride Nanotubes, Phys. Rev. Lett. 97, 085901 (2006).

[24] K. Chen et al., Ultrahigh thermal conductivity in isotopeenriched cubic boron nitride, Science 367, 555 (2020).

[25] L. Lindsay and D. Broido, Enhanced thermal conductivity and isotope effect in single-layer hexagonal boron nitride, Phys. Rev. B 84, 155421 (2011).

[26] H. Dong, P. Hirvonen, Z. Fan, and T. Ala-Nissila, Heat transport in pristine and polycrystalline single-layer hexagonal boron nitride, Phys. Chem. Chem. Phys. 20, 24602 (2018).

[27] C. Sevik, A. Kinaci, J. B. Haskins, and T. Çağın, Influence of disorder on thermal transport properties of boron nitride nanostructures, Phys. Rev. B 86, 075403 (2012).

[28] L. Lindsay and D. Broido, Theory of thermal transport in multilayer hexagonal boron nitride and nanotubes, Phys. Rev. B 85, 035436 (2012).

[29] S. Liu, R. He, L. Xue, J. Li, B. Liu, and J. H. Edgar, Single crystal growth of millimeter-sized monoisotopic hexagonal boron nitride, Chem. Mater. 30, 6222 (2018).

[30] See Supplemental Material at http://link.aps.org/ supplemental/10.1103/PhysRevLett.125.085902 for methods for sample preparation and theoretical calculations, additional sample characterizations, laser beam size and its effect on thermal conductivity, phonon dispersion and Gruneisen graphs, and thermal conductivities of $2-3 L$ isotopic BN.

[31] T. Taniguchi and K. Watanabe, Synthesis of high-purity boron nitride single crystals under high pressure by using Ba-BN solvent, J. Cryst. Growth 303, 525 (2007).

[32] I. Caretti and I. Jimenez, Point defects in hexagonal $\mathrm{BN}, \mathrm{BC}_{3}$ and $\mathrm{BC}_{x} N$ compounds studied by X-ray absorption near-edge structure, J. Appl. Phys. 110, 023511 (2011).

[33] L. H. Li, M. Petravic, B. C. C. Cowie, T. Xing, R. Peter, Y. Chen, C. Si, and W. H. Duan, High-resolution X-ray absorption studies of core excitons in hexagonal boron nitride, Appl. Phys. Lett. 101, 191604 (2012).

[34] L. H. Li, J. Cervenka, K. Watanabe, T. Taniguchi, and Y. Chen, Strong oxidation resistance of atomically thin boron nitride nanosheets, ACS Nano 8, 1457 (2014).

[35] Q. Cai, D. Scullion, A. Falin, K. Watanabe, T. Taniguchi, Y. Chen, E. J. G. Santos, and L. H. Li, Raman signature and phonon dispersion of atomically thin boron nitride, Nanoscale 9, 3059 (2017).

[36] B. R. Carvalho, Y. Hao, A. Righi, J. F. Rodriguez-Nieva, L. Colombo, R. S. Ruoff, M. A. Pimenta, and C. Fantini, 
Probing carbon isotope effects on the Raman spectra of graphene with different ${ }^{13} \mathrm{C}$ concentrations, Phys. Rev. B 92, 125406 (2015).

[37] J.-U. Lee, D. Yoon, H. Kim, S. W. Lee, and H. Cheong, Thermal conductivity of suspended pristine graphene measured by Raman spectroscopy, Phys. Rev. B 83, 081419 (2011).

[38] W. Cai, A. L. Moore, Y. Zhu, X. Li, S. Chen, L. Shi, and R. S. Ruoff, Thermal transport in suspended and supported monolayer graphene grown by chemical vapor deposition, Nano Lett. 10, 1645 (2010).

[39] S. Chen et al., Raman measurements of thermal transport in suspended monolayer graphene of variable sizes in vacuum and gaseous environments, ACS Nano 5, 321 (2011).

[40] R. Yan, J. R. Simpson, S. Bertolazzi, J. Brivio, M. Watson, X. Wu, A. Kis, T. Luo, A. R. Hight Walker, and H. Grace Xing, Thermal conductivity of monolayer molybdenum disulfide obtained from temperature-dependent Raman spectroscopy, ACS Nano 8, 986 (2014).

[41] D. Basko, S. Piscanec, and A. Ferrari, Electron-electron interactions and doping dependence of the two-phonon Raman intensity in graphene, Phys. Rev. B 80, 165413 (2009).
[42] N. Bonini, M. Lazzeri, N. Marzari, and F. Mauri, Phonon Anharmonicities in Graphite and Graphene, Phys. Rev. Lett. 99, 176802 (2007).

[43] A. K. Vallabhaneni, D. Singh, H. Bao, J. Murthy, and X. Ruan, Reliability of Raman measurements of thermal conductivity of single-layer graphene due to selective electron-phonon coupling: A first-principles study, Phys. Rev. B 93, 125432 (2016).

[44] A. Ward and D. J. P. R. B. Broido, Intrinsic phonon relaxation times from first-principles studies of the thermal conductivities of Si and Ge, Phys. Rev. B 81, 085205 (2010).

[45] Z. Tian, K. Esfarjani, J. Shiomi, A. S. Henry, and G. J. A. P. L. Chen, On the importance of optical phonons to thermal conductivity in nanostructures, Appl. Phys. Lett. 99, 053122 (2011).

[46] J. P. Perdew, J. A. Chevary, S. H. Vosko, K. A. Jackson, M. R. Pederson, D. J. Singh, and C. Fiolhais, Atoms, molecules, solids, and surfaces-applications of the generalized gradient approximation for exchange and correlation, Phys. Rev. B 46, 6671 (1992).

Correction: The affiliation for the 8th author contained an error and has been fixed. 